\title{
Dural sinus volume in children with syndromic craniosynostosis and intracranial hypertension
}

\author{
Robbin de Goederen, MD, ${ }^{1}$ Iris E. Cuperus, BSc, ${ }^{1}$ Robert C. Tasker, MBBS, MD, ${ }^{2}$ \\ Bianca K. den Ottelander, MD, ${ }^{1}$ Marjolein H. G. Dremmen, MD, PhD, ${ }^{3}$ \\ Marie-Lise C. van Veelen, MD, PhD, ${ }^{4}$ Jochem K. H. Spoor, MD, PhD, ${ }^{4}$ \\ Koen F. M. Joosten, MD, PhD, ${ }^{5}$ and Irene M. J. Mathijssen, MD, PhD ${ }^{1}$
}

\begin{abstract}
Departments of ${ }^{1}$ Plastic and Reconstructive Surgery, and Hand Surgery, ${ }^{3}$ Radiology, ${ }^{4}$ Neurosurgery, and ${ }^{5}$ Pediatrics, Intensive Care Unit, Erasmus MC, Rotterdam, The Netherlands; and '2Departments of Neurology and Anesthesia (Pediatrics), Harvard Medical School and Boston Children's Hospital, Boston, Massachusetts
\end{abstract}

\begin{abstract}
OBJECTIVE Intracranial hypertension is a major concern in children with syndromic craniosynostosis (sCS). Cerebral venous hypertension caused by cerebral venous outflow obstruction is believed to contribute to intracranial hypertension. The authors therefore hypothesized that cerebral venous volume would be increased in those children with sCS and intracranial hypertension.
\end{abstract}

METHODS In a case series of 105 children with sCS, of whom 32 had intracranial hypertension, cerebral MRI techniques were used to quantify the volume of the superior sagittal sinus, straight sinus (StrS), and both transverse sinuses.

RESULTS Linear regression showed that total cerebral venous volume increased by $580.8 \mathrm{~mm}^{3}$ per $\mathrm{cm}$ increase in occipitofrontal head circumference $(p<0.001)$. No significant difference was found between the intracranial hypertension group and the nonintracranial hypertension group $(p=0.470)$. Multivariate ANOVA showed increased StrS volume (as a proportion of total volume) in the intracranial hypertension group ( $8.5 \%$ vs $5.1 \%$ in the nonintracranial hypertension group, $\mathrm{p}<0.001)$. Multivariate logistic regression showed that a $100-\mathrm{mm}^{3}$ increase in StrS volume is associated with increased odds of having intracranial hypertension by $60 \%$ (OR 1.60, 95\% Cl 1.24-2.08).

CONCLUSIONS Although intracranial hypertension was not associated with total cerebral venous volume increase, it was associated with an isolated increase in StrS volume. Hence, it is unlikely that general cerebral venous outflow obstruction is the mechanism of intracranial hypertension in SCS. Rather, these findings indicate either a central cerebral vulnerability to intracranial hypertension or a mechanism involving venous blood redistribution.

https://thejns.org/doi/abs/10.3171/2019.12.PEDS19562

KEYWORDS craniosynostosis; cerebral venous circulation; dural sinus; intracranial hypertension; intracranial pressure; papilledema; craniofacial

$\mathrm{I}$ NTRACRANIAL hypertension is a major concern in children with syndromic craniosynostosis (sCS), and in Apert and Crouzon syndromes the incidence is as high as $53 \%$., 5 There are many complications of prolonged intracranial hypertension, with the most severe being irreversible visual loss. ${ }^{5}$ Intracranial hypertension may also impair neurodevelopment and impact behavior and learning. ${ }^{20,21}$

Initially, surgeons thought that limited growth of the cranial vault was the main reason for intracranial hypertension in children with craniosynostosis. Treatment focused primarily on increasing the intracranial volume. Over the years, more and more evidence showed that not only did reduced cranial growth affect the intracranial pressure (ICP), but also other factors such as obstructive sleep apnea (OSA) and cranial venous hypertension. , $^{90,12,25}$

Even currently, the mechanisms that account for intracranial hypertension in children with sCS are not fully understood, but anomalous cerebral venous drainage patterns are often found in such children. It appears that these anomalies may limit cerebral venous outflow and could thereby indirectly perturb CSF reabsorption. For example,

ABBREVIATIONS COS = confluence of the dural sinuses; FOHR = frontooccipital horn ratio; ICP = intracranial pressure; IIH = idiopathic intracranial hypertension; MANOVA = multivariate ANOVA; OCT = optical coherence tomography; OFC = occipitofrontal head circumference; OSA = obstructive sleep apnea; sCS = syndromic craniosynostosis; SSS = superior sagittal sinus; StrS = straight sinus; TS = transverse sinus.

SUBMITTED September 24, 2019. ACCEPTED December 3, 2019.

INCLUDE WHEN CITING Published online January 31, 2020; DOI: 10.3171/2019.12.PEDS19562. 
many patients with sCS have absent or stenotic cerebral venous sinuses-especially of the transverse sinus (TS), sigmoid sinus, and jugular complex ${ }^{4,13,28}$ - that are related to an underlying genetic mutation. ${ }^{29}$ These patients also have venous drainage through big emissary veins near the occiput and, in some cases, these emissary veins are the main drainage system of the brain., $4,13,28$

Another condition that is somewhat similar to intracranial hypertension in craniosynostosis is idiopathic intracranial hypertension (IIH). Patients suffering from IIH experience symptoms from intracranial hypertension such as disabling headaches and papilledema. Their ICP is indeed increased, but without an identifiable cause..$^{18}$ Over the years, researchers have found that many patients with IIH have increased ICP due to TS stenosis. ${ }^{6}$ These stenoses can be resolved by stenting the sinus or by performing a lumbar puncture. In a study by Rohr et al., the authors measured the dural sinus volumes before and after lumbar puncture. ${ }^{24}$ They reported that patients with IIH had significantly smaller sinus volumes than the healthy control group. Upon normalization of the ICP, the sinus volumes increased significantly, independent from the disappearing TS stenosis.

Taking these findings together, it is unknown to what extent venous anomalies contribute to the development of intracranial hypertension in children with sCS. The hypothesis that a narrower jugular foramen (and thereby restricted outflow of the sinuses) causes intracranial hypertension in children with sCS is controversial., ${ }^{3,22,28}$ As a first step in examining this venous obstruction hypothesis in children with sCS we tested whether intracranial hypertension is associated with a change in dural sinus volumes.

\section{Methods \\ Patients}

This study is part of an ongoing national clinical program for children with sCS or complex craniosynostosis (multiple-suture craniosynostosis without a known genetic mutation) treated at the Dutch Craniofacial Centre (Sophia Children's Hospital, Erasmus University Medical Center, Rotterdam, The Netherlands). The study was approved by our institutional research ethics committee for human studies and all children are managed according to a previously described protocol. ${ }^{26}$ In this report, we focus on cerebral MRI scans performed between 2008 and 2018. All children who had undergone a 3D T2-weighted scan with isometric voxels of the brain were eligible for inclusion, and were then split into two groups based on the presence or absence of intracranial hypertension. Of 346 children with $\mathrm{sCS}$ being treated in our center during the prespecified time period, 105 children had an eligible MR image. Hence, we have included MRI scans of 32 children in the intracranial hypertension group and 73 children in the nonintracranial hypertension group.

\section{Intracranial Hypertension}

As described elsewhere, ${ }^{26}$ the presence of intracranial hypertension in our clinical protocol was based on findings that included funduscopic tests, optical coherence tomography (OCT) (Spectralis OCT scanner, Heidelberg
Engineering), invasive ICP measurements, and the size of the cerebral ventricles on MRI. Hydrocephalus was diagnosed when the enlargement of the ventricles was progressive on two consecutive MRI or CT scans, or if the frontooccipital horn ratio (FOHR) was $>0.44$. Only patients with untreated hydrocephalus were included in this part of the study. Children were scored as having intracranial hypertension when they had papilledema, hydrocephalus, a positive invasive ICP measurement, or an increased total retina thickness on OCT. All children underwent funduscopic tests at least on a yearly basis and more frequently if there was clinical suspicion of intracranial hypertension.

\section{MRI Acquisition}

All MRI scans were performed on a 1.5-T scanner (MR Signa Excite HD, GE Healthcare) and the imaging protocol included a 3D sagittal T2-weighted MRI sequence with isometric voxels. The imaging parameters were slice thickness $1.6 \mathrm{~mm}$, no slice gap, field of view $20 \mathrm{~cm}$, matrix size $224 \times 224$, TE $90 \mathrm{msec}$, and TR $2500 \mathrm{msec}$. Infants and small children who were unable to lie still during the MRI scan were scanned under general anesthesia, as per the clinical algorithm.

In the current clinical protocol for sCS, all children are scheduled for a brain MRI scan shortly after their first visit to our center and at age 4. Children with Apert or Crouzon syndrome undergo additional MRI at 2 years of age. When the neurosurgeon suspects intracranial hypertension or tonsillar herniation, an additional MRI scan may be performed. The 3D T2-weighted sequence with isometric voxels was added to the scanning protocol in 2008, and therefore not all of our patients with sCS have undergone such a scan.

\section{Segmentation of Dural Sinuses}

MRI scans in DICOM format were imported into the 3D Slicer program ${ }^{7}$ (version 4.8.0) and the Editor tool was used for cerebral venous sinus segmentation. Three-dimensional reconstructions of the dural sinuses were made and measurements taken in all anatomical planes. Sinuses of interest included the superior sagittal sinus (SSS), the straight sinus (StrS), and both TSs (the sigmoid sinus was included with the TS volume). The crista galli was defined as the initial point of the SSS, and the confluence of the dural sinuses $(\mathrm{CoS})$ was considered the end point. In the StrS, the confluence of the great cerebral vein and the inferior sagittal sinus was defined as the starting point and the $\mathrm{CoS}$ the end point. The CoS was considered the initial point of the TS and the jugular foramen its end point. All segmentations were performed by one researcher (I.E.C.) who was blinded to the presence or absence of intracranial hypertension in the patient. A second independent researcher (R.D.G.) checked the segmentation, also while blinded to the intracranial hypertension status of the subject. To determine reproducibility of the segmentation, the scans of 10 randomly selected patients were segmented again after a few months by the second researcher. The intraclass correlation coefficient was calculated using the total volume only. Once consensus on the segmentation was achieved, a 3D model of the segmented sinuses was 
created and the total sinus volume was calculated in cubic millimeters $\left(\mathrm{mm}^{3}\right)$.

The two researchers divided the segmented volume of all the sinuses by consensus. The SSS was cleaved superiorly to the CoS, the StrS anteriorly to the CoS, and the TSs medially in the CoS. Finally, the split 3D model was made (Fig. 1) and each separate volume of interest was calculated. The TS with the greater volume was considered the dominant TS, and the one with the lower volume the nondominant TS. Furthermore, all children were evaluated on the presence of occipital emissary veins based on their MRI scan together with contrast-enhanced CT scans if present. Patients were scored as having occipital emissary veins when, upon visual inspection, one of the veins had a bigger diameter than approximately $33 \%$ of the diameter of the distal part of the SSS.

\section{Statistical Analysis}

Statistical analyses were performed using the statistical programming language $\mathrm{R}$ (version 3.6.0, $\mathrm{R}$ Foundation for Statistical Computing). All data were checked for normality and nonnormally distributed data were log-transformed when needed. Simple between-group comparisons were conducted using the Student t-test for unpaired data. Linear regression analysis was performed to evaluate the effect of age, occipitofrontal head circumference (OFC), and intracranial hypertension on the total cerebral volume. To compare the total difference in individual dural sinus volumes (i.e., SSS volume, StrS volume, TS dominant volume, and TS nondominant volume) between the intracranial hypertension and the nonintracranial hypertension group, a multivariate ANOVA (MANOVA) was performed. In this analysis, the dural sinus volumes were presented as a proportion of the total volume. To evaluate the effect size of the significant differences found in the MANOVA, a multivariate logistic regression was performed, correcting for age and OFC z-score.

\section{Results}

Patient characteristics and mean sinus volumes per group are presented in Table 1. As expected, patients with intracranial hypertension more frequently showed occipital emissary veins than the nonintracranial hypertension group (65.6\% vs 39.7\%). Table 2 shows further details on the exact diagnostic criteria used for the diagnosis of intracranial hypertension. Figure 2 shows scatterplots of the total sinus volumes set on an $\mathrm{x}$-axis of age, with a fitted linear regression line based on the presence of intracranial hypertension; age was log-transformed for the regression due to a nonnormal distribution. The same method was performed for the total volumes against the OFC. The intraclass correlation coefficient was 0.99 (95\% CI 0.971.00). Linear regression showed that OFC had the biggest impact on total volume with an increase of $580.8 \mathrm{~mm}^{3}$ per $\mathrm{cm}(\mathrm{p}<0.001) . \log ($ age $)$ and intracranial hypertension had no significant effect on the total volume $(\beta 2486.8$ [p $=0.219]$ and $\beta-108.4[p=0.843]$, respectively). After normalizing the total volumes by dividing them by OFC, an unpaired Student t-test showed no significant difference between the intracranial hypertension group and the nonintracranial hypertension group $(\mathrm{p}=0.470)$.
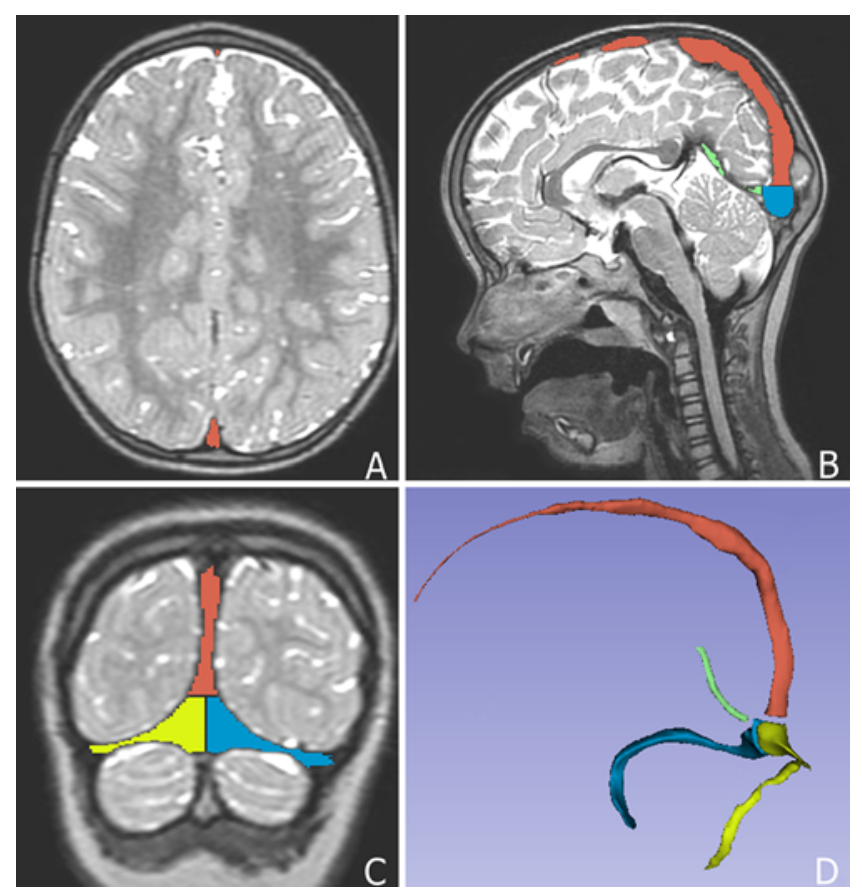

FIG. 1. T2-weighted MR images showing the segmentation of the dural sinus volumes. Red represents the SSS, green the StrS, and yellow and blue the TSs. A: Coronal image. B: Midsagittal image. C: Axial image at the level of the confluence of sinuses. D: Three-dimensional image of the segmented sinuses. Figure is available in color online only.

A MANOVA comparing the combination of individual sinus volumes between the two groups showed a significant difference (Pillai's trace 0.208, $\mathrm{p}<0.001$ ). The post hoc analysis of the MANOVA showing the influence of the individual dural sinus volumes is presented in Table 3. The results show that the difference between the groups is caused by an increased StrS volume proportion of the total volume in the intracranial hypertension group, i.e., $8.5 \%$ versus $5.1 \%$ in the nonintracranial hypertension group $(\mathrm{p}$ $<0.001$; Fig. 3). All other volumes were not significantly different between the groups. An example of a clear increase of the StrS on an MR image of a child with Crouzon syndrome and intracranial hypertension is shown in Fig. 4.

Multivariate logistic regression analysis of the statistical predictive value of the StrS volume on the presence of intracranial hypertension, corrected for age and OFC z-score, was then performed. The analysis showed that an StrS volume increase of $100 \mathrm{~mm}^{3}$ had an OR of $1.60(95 \%$ CI 1.24-2.08). This indicates that the odds of having intracranial hypertension increases by $60 \%$ with every 100 $\mathrm{mm}^{3}$ increase in StrS volume.

\section{Discussion}

This study in children with sCS shows that dural sinus volumes are strongly correlated with head circumference. There was no difference in total volume between the intracranial hypertension group and the nonintracranial hypertension group. However, there was a measurable increase of the StrS volume in the presence of intracranial 
TABLE 1. Characteristics of the total study population of children with sCS according to intracranial hypertension

\begin{tabular}{|c|c|c|}
\hline Variable & $\begin{array}{c}\text { Intracranial Hypertension } \\
\text { Group }\end{array}$ & $\begin{array}{c}\text { Nonintracranial Hypertension } \\
\text { Group }\end{array}$ \\
\hline No. of patients & 32 & 73 \\
\hline Median age (IQR), yrs & $2.44(0.64-4.08)$ & $3.97(0.62-6.15)$ \\
\hline Males/females & $23 / 9$ & $29 / 44$ \\
\hline Mean OFC (range), cm & $47.1(36.0-54.0)$ & $48.5(38.0-60.0)$ \\
\hline Mean OFC z-score (range) & $-0.25(-3.0$ to 2.63$)$ & $0.001(-3.32$ to 3.23$)$ \\
\hline \multicolumn{3}{|l|}{ Diagnosis, n (\%) } \\
\hline Apert syndrome & $6(18.8)$ & $15(20.5)$ \\
\hline Crouzon syndrome & $15(46.9)$ & $23(31.5)$ \\
\hline Muenke syndrome & $0(0)$ & $11(15.1)$ \\
\hline Saethre-Chotzen syndrome & $4(12.5)$ & $7(9.6)$ \\
\hline TCF12 mutation & $0(0)$ & $6(8.2)$ \\
\hline Complex craniosynostosis & $7(21.9)$ & $11(15.1)$ \\
\hline Mean FOHR (range) & $0.39(0.21-0.68)$ & $0.34(0.26-0.53)$ \\
\hline Hydrocephalus, n (\%) & $8(25)$ & $0(0)$ \\
\hline Anesthesia, $n(\%)^{*}$ & $31(96.9)$ & $56(76.7)$ \\
\hline Operated, $\mathrm{n}(\%) \dagger$ & $19(59.4)$ & $49(67.1)$ \\
\hline Occipital emissary veins, $\mathrm{n}(\%)$ & $21(65.6)$ & $29(39.7)$ \\
\hline Mean total sinus vol (range), $\mathrm{mm}^{3}$ & $7663.4(1930.1-20593.5)$ & $3922.1(786.5-7161.4)$ \\
\hline Mean SSS vol (range), $\mathrm{mm}^{3}$ & $3922.1(786.5-7161.4)$ & $4175.0(1030.7-9107.8)$ \\
\hline Mean StrS vol (range), $\mathrm{mm}^{3}$ & $553.8(84.4-1080.4)$ & $404.3(61.4-1030.9)$ \\
\hline Mean TS dominant vol (range), $\mathrm{mm}^{3}$ & $2157.4(284.3-6386.1)$ & $2561.0(511.7-7760.5)$ \\
\hline Mean TS nondominant vol (range), $\mathrm{mm}^{3}$ & $969.3(0-6062.9)$ & $1167.6(42.0-4060.86)$ \\
\hline
\end{tabular}

TABLE 2. Characteristics of intracranial hypertension diagnostic criteria

\begin{tabular}{lc}
\hline \multicolumn{1}{c}{ Diagnostic Variable } & No. \\
\hline Method of diagnosing intracranial hypertension & 32 \\
\hline Papilledema & 27 \\
\hline Hydrocephalus & 9 \\
\hline Invasive ICP measurement & 3 \\
\hline OCT & 1 \\
\hline Specification & 19 \\
\hline Only papilledema & 3 \\
\hline Papilledema + positive ICP measurement & 4 \\
\hline Hydrocephalus + papilledema & 1 \\
\hline Papilledema + positive OCT & 5 \\
\hline Only hydrocephalus &
\end{tabular}

The top half of the table represents the total count of occurring events; the bottom half provides specifics on the combination of events occurring in the same patients. hypertension, while in the other sinuses this was not the case. Moreover, every $100-\mathrm{mm}^{3}$ increase in StrS volume increases the odds of having intracranial hypertension by $60 \%$. Hence the results of this study show cerebral blood volume redistribution in children with intracranial hypertension, but it is unclear how the regional volume changes in StrS are explained.

Intracranial hypertension in $\mathrm{sCS}$ may be caused by 1 of 4 contributors: craniocerebral disproportion, venous hypertension, CSF outflow obstruction, and OSA. For each contributor, we will discuss their possible relation to an increase in dural sinus volumes. The first contributor to intracranial hypertension is craniocerebral disproportion. In a previous study, we showed that stagnating head growth was the most important predictor of developing intracranial hypertension. ${ }^{26}$ In the Monro-Kellie doctrine, ${ }^{31}$ if skull growth is impaired, there is competition among brain growth, cerebral blood volume, and CSF volume. As a consequence, the arterial blood pressure and arterial inflow to the brain are increased to preserve the cerebral perfusion. ${ }^{11,19}$ For example, in a previous study, we showed that children with sCS and intracranial hypertension had higher transcranial Doppler flow velocity in the middle cerebral artery, which normalized after cranial vault expansion. ${ }^{27}$ An increase in arterial flow also means 
TABLE 3. MANOVA of dural sinus volumes in the intracranial versus nonintracranial hypertension groups

\begin{tabular}{lccrc}
\hline \multicolumn{1}{c}{ Variable } & $\begin{array}{c}\text { Intracranial Hypertension } \\
\text { (mean, SD) }\end{array}$ & $\begin{array}{c}\text { Nonintracranial Hypertension } \\
\text { (mean, SD) }\end{array}$ & F Statistic & p Value \\
\hline SSS vol, \% & $53.6(9.3)$ & $51.4(7.5)$ & 0.88 & 0.350 \\
\hline StrS vol, \% & $8.5(4.6)$ & $5.1(2.2)$ & 25.71 & $<0.001^{*}$ \\
\hline TS dominant, \% & $26.1(8.3)$ & $29.0(7.4)$ & 2.66 & 0.106 \\
\hline TS nondominant, \% & $10.8(7.7)$ & $13.5(5.8)$ & 3.29 & 0.073 \\
\hline
\end{tabular}

Mean dural sinus volumes, presented as a proportion of the total volume, for the intracranial versus nonintracranial hypertension groups. The presented $p$ value is the result of a post hoc analysis of the MANOVA.

* Statistically significant.

an increase in central cerebral venous flow, which could explain the increased volume of the StrS observed in the current study.

The second contributor to intracranial hypertension is venous hypertension, or venous outflow obstruction. Children with sCS can have many cerebral venous malformations, including aplastic TS, abundant emissary veins, and malformations of the jugular/sigmoid complex. . $^{8,17,28,29}$ Most of the malformations could potentially cause an obstruction of cerebral venous outflow. Sainte-Rose et al. confirmed that venous obstruction caused intracranial hypertension in some children. ${ }^{25}$ Venous bypassing of the obstruction successfully resolved the intracranial hypertension in one of these children. An increase in StrS volume raises the suspicion of venous outflow obstruction distally to the StrS as a possible cause of the intracranial hypertension in these children. However, the results of our study do not provide any additional clues to the site of an obstruction.

The third known contributor to intracranial hypertension is CSF outflow obstruction. The most common consequence of CSF outflow obstruction is ventriculomegaly and ultimately hydrocephalus. The size of the ventricles is easily quantified in this study by measuring the FOHR. In this study, the size of the FOHR was not associated with dural sinus volumes. In an exploratory analysis (data not presented), we accounted for the interaction between FOHR and intracranial hypertension and found that increased StrS volume was solely attributed to intracranial hypertension. Eight children in this study had untreated hydrocephalus at the time of MRI, but this condition was not related to any changes in sinus volumes.

The last contributor to intracranial hypertension, OSA, is unlikely to be related to any volumetric changes in dural sinuses. OSA increases the ICP by intracerebral vasodilatation caused by hypercapnia when apneas occur, which is mostly during REM sleep..$^{14}$ During the present study, the patients were either awake or intubated, and therefore did not have apneas during MRI. In addition, exploratory statistical analysis showed that OSA did not have an effect on sinus volumes (data not presented).

Of further interest was the difference in effect of intracranial hypertension between the StrS and the other dural sinuses. At the moment, it is unclear how this difference can be explained and the current data do not provide the answers to this question. However, two theories could ex- plain this difference. The first theory involves the positioning of the different dural sinuses and their direct surroundings. The sinuses of the superficial cerebral venous system (SSS and TS) are located directly adjacent to bone of the skull. ${ }^{15,30}$ Surgeons in our center report exaggerated thinning of the bone at the location of the superficial dural sinuses, which seems to confirm that these sinuses compress the adjacent skull. The StrS, however, is part of the deep cerebral venous system and is located in the tentorium. While pressure increases in all dural sinuses, the StrS, not being restricted by bone, could be the one that
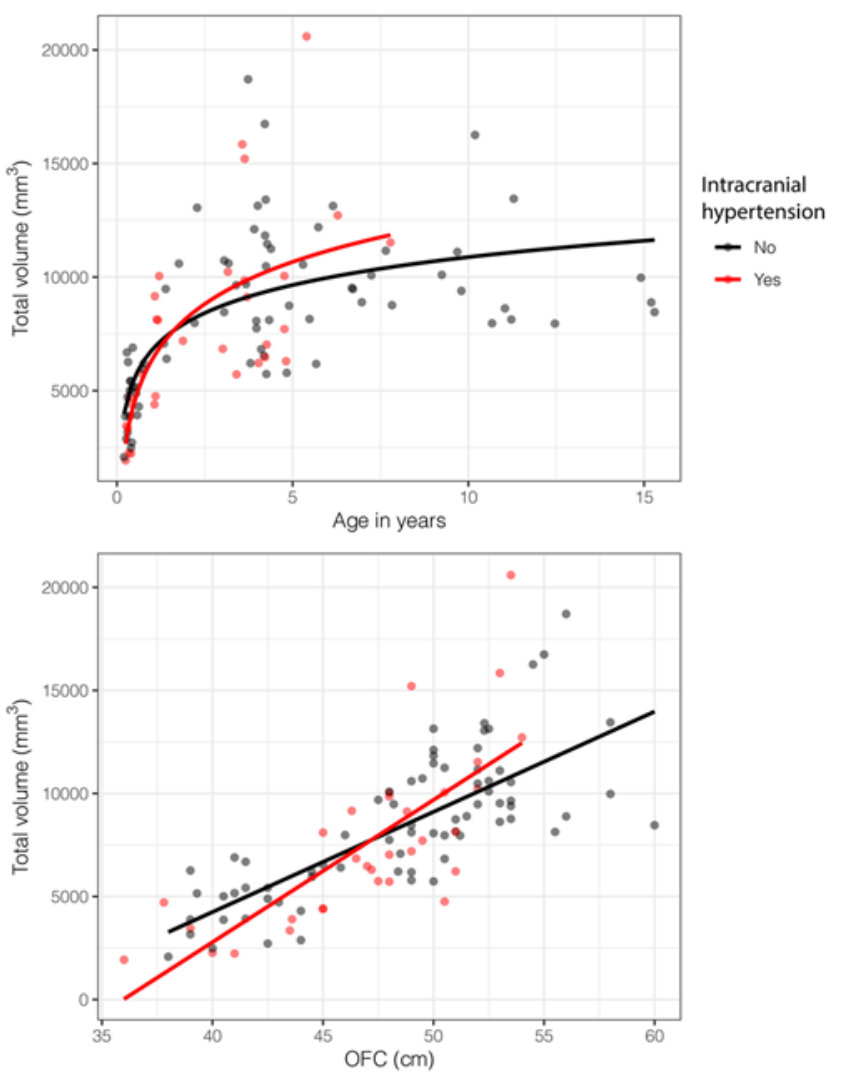

FIG. 2. Scatterplots of the total sinus volumes against age (upper) and OFC (lower), with a fitted regression line per group. Age was logtransformed in the fitted regression line. Figure is available in color online only. 

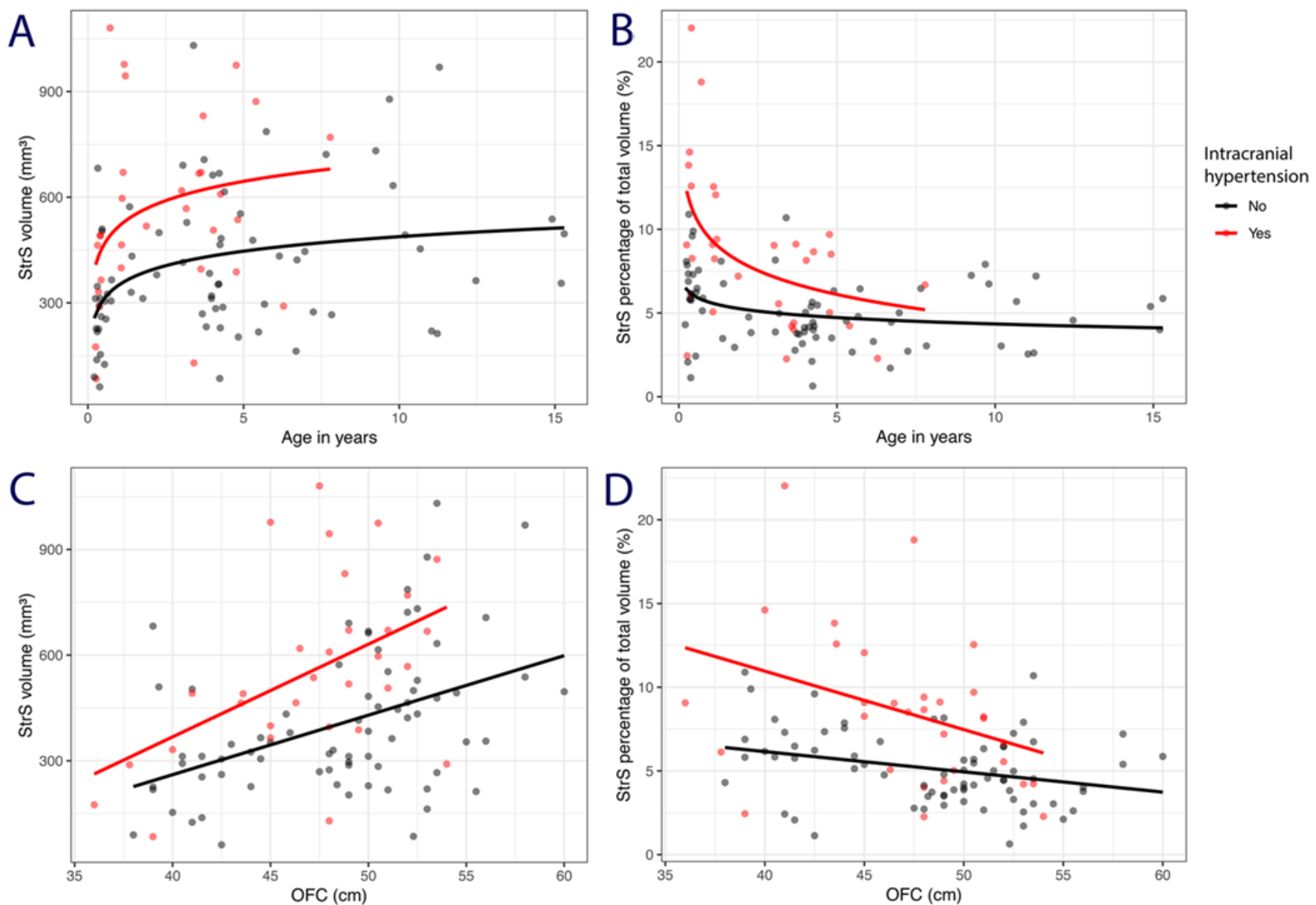

FIG. 3. Scatterplots of the StrS against age and OFC, with a fitted regression line per group. Panels $\mathbf{A}$ and $\mathbf{C}$ represent the absolute volumes, while panels $\mathbf{B}$ and $\mathbf{D}$ represent the StrS as proportions of the total volume. Age was log-transformed in the fitted regression lines. Figure is available in color online only.

expands most easily, possibly explaining why the volume of the StrS increases and the volumes of the other sinuses do not. The second theory is related to the emissary veins connecting the superficial intracerebral venous system to the extracerebral venous system, together with the theory of increased flow in cases of intracranial hypertension. The superficial cerebral venous system has emissary veins through the skull, ${ }^{1,2,16}$ through which blood can exit the intracranial space in cases of increased blood flow, while blood from the deep veins can only travel through the StrS to the superficial system. ${ }^{1}$ The higher flow and pressures in the SSS at the level of the CoS might cause a relative obstruction for the StrS, increasing its volume. Taking all of the above together, the most prudent hypothesis for the association between intracranial hypertension and increased StrS but normal total dural sinus volume is either a redistribution in regional cerebral blood flow (i.e., away from the superficial cortical structures and toward the central gray matter) or the rerouting of blood through the emissary veins and avoiding regular superficial structures in venous drainage, which is not possible for the deeper central tissue.

This study has some limitations. The first limitation is that our MRI scans were not contrast-enhanced. Contrast enhancement could increase the accuracy of the volume segmentation. However, this factor was probably of minor influence because we used identical methods for both children with and without intracranial hypertension with a high reproducibility. Second, the children in our study most probably did not have a peak in ICP during the scan, because the peaks of ICP in sCS are mostly REM sleep related. ${ }^{32}$ Therefore, we were not able to account for the dynamic aspects of intracranial hypertension. However, even with this suboptimal timing of imaging, we still found an increase of the StrS volume. Even though the StrS volume is less than $5 \%$ of the total volume, the increase in volume is remarkable. Future studies could focus on dynamic imaging to measure volumes and flow velocities during REM sleep. The third limitation is the variable timing of the eligible MR images. Given the limited availability of the needed MRI sequence, we chose to include a wide range of ages and correct for age or OFC in the statistical analyses afterwards. This approach made it impossible to properly investigate the effect of surgery on the sinus volumes, something that could be accomplished in future research. The fourth limitation was the fact that we used OFC as a proxy for intracranial volume. The intracranial volume was difficult to measure on the 3D T2-weighted MRI se- 


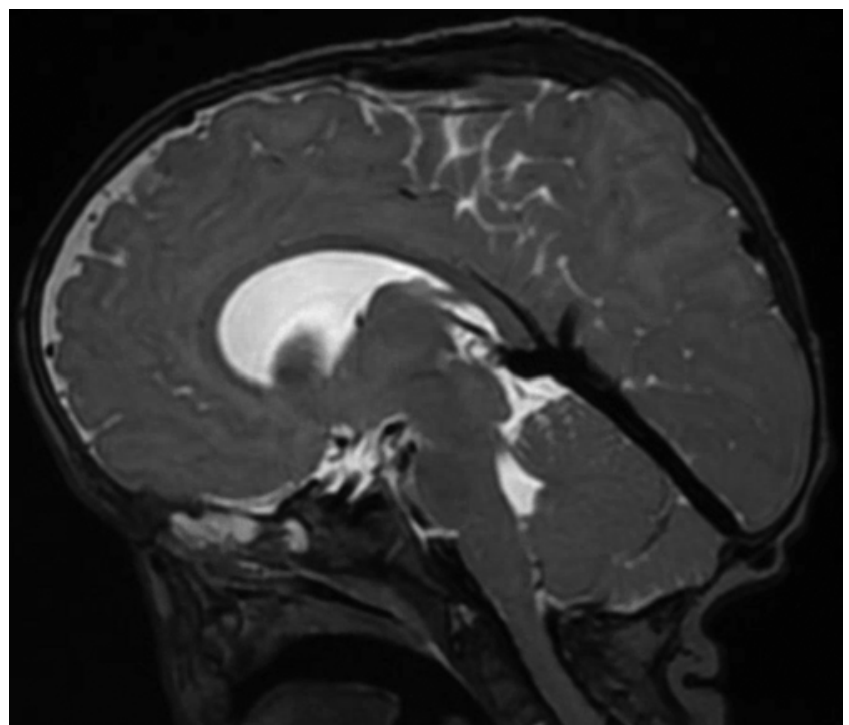

FIG. 4. Midsagittal T2-weighted MR image of a child with Crouzon syndrome and intracranial hypertension, showing a marked distension of the StrS.

quence we used in this study. This was mostly because of the poor visualization of bone on this sequence. We believe OFC to be a useful proxy, as we have verified this in one of our previous studies. ${ }^{23}$ The fifth limitation was the limited availability of the 3D T2-weighted MRI sequence with isometric voxels that we used in this study. Only onethird of the patients had such a scan available following its introduction in the scanning protocol in 2008. Thus, a lot of children who already had an MRI scan did not have the correct sequence available. Therefore, there might have been a selection bias that could influence the generalizability of the results. Lastly, we did not perform invasive ICP measurements in all patients, but relied mostly on funduscopy to diagnose intracranial hypertension. This might result in a false-negative outcome and missing episodes of intracranial hypertension. Given the long-term and frequent follow-up of our patients, the impact of these missed cases is probably limited.

\section{Conclusions}

This study shows that in children with sCS, intracranial hypertension is associated with increased StrS volume, but total cerebral venous volume is unchanged. In our view, these findings rule out a focal restriction of dural sinus flow as a cause of intracranial hypertension, and may suggest a mechanism of venous blood redistribution. However, further research should focus on dynamic studies of regional cerebral blood flow and venous flow in order to explore venous blood volume redistribution.

\section{Acknowledgments}

This study was funded by Stichting Hoofdzaak, a foundation that collects donations for research for patients with craniofacial disorders.

\section{References}

1. Andeweg J: The anatomy of collateral venous flow from the brain and its value in aetiological interpretation of intracranial pathology. Neuroradiology 38:621-628, 1996

2. Baltsavias G, Parthasarathi V, Aydin E, Al Schameri RA, Roth P, Valavanis A: Cranial dural arteriovenous shunts. Part 1. Anatomy and embryology of the bridging and emissary veins. Neurosurg Rev 38:253-264, 2015

3. Booth CD, Figueroa RE, Lehn A, Yu JC: Analysis of the jugular foramen in pediatric patients with craniosynostosis. $\mathbf{J}$ Craniofac Surg 22:285-288, 2011

4. Copeland AE, Hoffman CE, Tsitouras V, Jeevan DS, Ho ES, Drake JM, et al: Clinical significance of venous anomalies in syndromic craniosynostosis. Plast Reconstr Surg Glob Open 6:e1613, 2018

5. de Jong T, Bannink N, Bredero-Boelhouwer HH, van Veelen ML, Bartels MC, Hoeve LJ, et al: Long-term functional outcome in 167 patients with syndromic craniosynostosis; defining a syndrome-specific risk profile. J Plast Reconstr Aesthet Surg 63:1635-1641, 2010

6. Farb RI, Vanek I, Scott JN, Mikulis DJ, Willinsky RA, Tomlinson G, et al: Idiopathic intracranial hypertension: the prevalence and morphology of sinovenous stenosis. Neurology 60:1418-1424, 2003

7. Fedorov A, Beichel R, Kalpathy-Cramer J, Finet J, FillionRobin JC, Pujol S, et al: 3D Slicer as an image computing platform for the Quantitative Imaging Network. Magn Reson Imaging 30:1323-1341, 2012

8. Florisson JM, Barmpalios G, Lequin M, van Veelen ML, Bannink N, Hayward RD, et al: Venous hypertension in syndromic and complex craniosynostosis: the abnormal anatomy of the jugular foramen and collaterals. J Craniomaxillofac Surg 43:312-318, 2015

9. Gault DT, Renier D, Marchac D, Jones BM: Intracranial pressure and intracranial volume in children with craniosynostosis. Plast Reconstr Surg 90:377-381, 1992

10. Gonsalez S, Hayward R, Jones B, Lane R: Upper airway obstruction and raised intracranial pressure in children with craniosynostosis. Eur Respir J 10:367-375, 1997

11. Guild SJ, Saxena UA, McBryde FD, Malpas SC, Ramchandra R: Intracranial pressure influences the level of sympathetic tone. Am J Physiol Regul Integr Comp Physiol 315:R1049-R1053, 2018

12. Hayward R: Venous hypertension and craniosynostosis. Childs Nerv Syst 21:880-888, 2005

13. Jeevan DS, Anlsow P, Jayamohan J: Abnormal venous drainage in syndromic craniosynostosis and the role of CT venography. Childs Nerv Syst 24:1413-1420, 2008

14. Jennum P, Børgesen SE: Intracranial pressure and obstructive sleep apnea. Chest 95:279-283, 1989

15. Kiliç T, Akakin A: Anatomy of cerebral veins and sinuses. Front Neurol Neurosci 23:4-15, 2008

16. Kim LK, Ahn CS, Fernandes AE: Mastoid emissary vein: anatomy and clinical relevance in plastic \& reconstructive surgery. J Plast Reconstr Aesthet Surg 67:775-780, 2014

17. Martinez-Perez D, Vander Woude DL, Barnes PD, Scott RM, Mulliken JB: Jugular foraminal stenosis in Crouzon syndrome. Pediatr Neurosurg 25:252-255, 1996

18. Mollan SP, Markey KA, Benzimra JD, Jacks A, Matthews TD, Burdon MA, et al: A practical approach to, diagnosis, assessment and management of idiopathic intracranial hypertension. Pract Neurol 14:380-390, 2014

19. Paton JF, Dickinson CJ, Mitchell G: Harvey Cushing and the regulation of blood pressure in giraffe, rat and man: introducing 'Cushing's mechanism.' Exp Physiol 94:11-17, 2009

20. Renier D, Lajeunie E, Arnaud E, Marchac D: Management of craniosynostoses. Childs Nerv Syst 16:645-658, 2000

21. Renier D, Sainte-Rose C, Marchac D, Hirsch JF: Intracranial pressure in craniostenosis. J Neurosurg 57:370-377, 1982 
22. Rich PM, Cox TC, Hayward RD: The jugular foramen in complex and syndromic craniosynostosis and its relationship to raised intracranial pressure. AJNR Am J Neuroradiol 24:45-51, 2003

23. Rijken BF, den Ottelander BK, van Veelen ML, Lequin MH, Mathijssen IM: The occipitofrontal circumference: reliable prediction of the intracranial volume in children with syndromic and complex craniosynostosis. Neurosurg Focus 38(5):E9, 2015

24. Rohr A, Bindeballe J, Riedel C, van Baalen A, Bartsch T, Doerner L, et al: The entire dural sinus tree is compressed in patients with idiopathic intracranial hypertension: a longitudinal, volumetric magnetic resonance imaging study. Neuroradiology 54:25-33, 2012

25. Sainte-Rose C, LaCombe J, Pierre-Kahn A, Renier D, Hirsch JF: Intracranial venous sinus hypertension: cause or consequence of hydrocephalus in infants? J Neurosurg 60:727736,1984

26. Spruijt B, Joosten KF, Driessen C, Rizopoulos D, Naus NC, van der Schroeff MP, et al: Algorithm for the management of intracranial hypertension in children with syndromic craniosynostosis. Plast Reconstr Surg 136:331-340, 2015

27. Spruijt B, Tasker RC, Driessen C, Lequin MH, van Veelen ML, Mathijssen IM, et al: Abnormal transcranial Doppler cerebral blood flow velocity and blood pressure profiles in children with syndromic craniosynostosis and papilledema. J Craniomaxillofac Surg 44:465-470, 2016

28. Taylor WJ, Hayward RD, Lasjaunias P, Britto JA, Thompson DN, Jones BM, et al: Enigma of raised intracranial pressure in patients with complex craniosynostosis: the role of abnormal intracranial venous drainage. J Neurosurg 94:377-385, 2001

29. Tischfield MA, Robson CD, Gilette NM, Chim SM, Sofela FA, DeLisle MM, et al: Cerebral vein malformations result from loss of Twist1 expression and BMP signaling from skull progenitor cells and dura. Dev Cell 42:445-461.e5, 2017
30. Uddin MA, Haq TU, Rafique MZ: Cerebral venous system anatomy. J Pak Med Assoc 56:516-519, 2006

31. Wilson MH: Monro-Kellie 2.0: The dynamic vascular and venous pathophysiological components of intracranial pressure. J Cereb Blood Flow Metab 36:1338-1350, 2016

32. Yokota A, Matsuoka S, Ishikawa T, Kohshi K, Kajiwara H: Overnight recordings of intracranial pressure and electroencephalography in neurosurgical patients. Part II: Changes in intracranial pressure during sleep. J UOEH 11:383-391, 1989

\section{Disclosures}

The authors report no conflict of interest concerning the materials or methods used in this study or the findings specified in this paper.

\section{Author Contributions}

Conception and design: de Goederen, Cuperus, Dremmen, Mathijssen. Acquisition of data: de Goederen, Cuperus, den Ottelander, Mathijssen. Analysis and interpretation of data: de Goederen, Cuperus, Tasker, van Veelen, Spoor. Drafting the article: de Goederen, Cuperus. Critically revising the article: Tasker, Dremmen, van Veelen, Spoor, Joosten, Mathijssen. Reviewed submitted version of manuscript: Cuperus, Tasker, den Ottelander, Dremmen, van Veelen, Spoor, Joosten, Mathijssen. Statistical analysis: de Goederen, Tasker. Administrative/technical/material support: den Ottelander, Dremmen. Study supervision: Joosten, Mathijssen.

\section{Correspondence}

Robbin de Goederen: Erasmus MC, Rotterdam, The Netherlands. r.degoederen@erasmusmc.nl. 